\title{
Detection of a new reaction by-product in BDDE cross-linked autoclaved hyaluronic acid hydrogels by LC-MS analysis
}

This article was published in the following Dove Press journal: Medical Devices: Evidence and Research

\author{
Javier Fidalgo* \\ Pierre-Antoine Deglesne* \\ Rodrigo Arroyo* \\ Lilian Sepúlveda* \\ Evgeniya Ranneva \\ Philippe Deprez
}

Scientific Department, Skin Tech Pharma Group, Castello D'Empúries, Cataluña, Spain

*These authors contributed equally to this work
Correspondence: Philippe Deprez Scientific Department, Skin Tech Pharma Group, C/ Pla de l'Estany, 29 (Pol. Industrial "El Pla"), Castelló d'Empúries 17486, Cataluña, Spain Email phd@estetik.com
Background: Hyaluronic acid (HA), a naturally occurring polysaccharide, is used in the production of dermal fillers for esthetic purposes. As it has a few days of half-life in human tissues, HA-based dermal filler is chemically modified to increase its lifetime in the body. The most common modification used in commercial HA-based filler is the cross-linking of HA chains using 1,4-butanediol diglycidyl ether (BDDE) as cross-linking agent. Residual, or unreacted, BDDE is considered nontoxic when it is $<2$ parts per million (ppm); therefore, the quantification of residual BDDE in the final dermal filler is mandatory to ensure the safety of the patients.

Materials and methods: The present study describes the detection and characterization of one by-product of the cross-linking reaction between BDDE and HA in alkaline conditions by combining both liquid chromatography and mass spectroscopy (LC-MS).

Results: After different analyses, it was found that the alkaline conditions and the high temperatures employed to sterilize the HA-BDDE hydrogel promote the formation of this new by-product, a "propene glicol-like" compound. LC-MS analysis confirmed that this by-product have the same monoisotopic mass as that of BDDE, a different retention time (tR), and also a different UV absorbance ( $\lambda=200 \mathrm{~nm}$ ) pattern. Unlike BDDE, it was observed in the LC-MS analysis that this by-product had a higher detection at $200 \mathrm{~nm}$ in the same assay conditions.

Conclusion: These results suggest that this new compound does not have an epoxide on its structure. The discussion is open to assess the risk of this new by-product found in the production of HA-BDDE hydrogels (HA dermal fillers) for commercial purposes.

Keywords: hyaluronic acid, HA dermal fillers, cross-linked hyaluronic acid, BDDE, LC-MS analysis, BDDE by-product.

\section{Introduction}

Hyaluronic acid (HA)-based fillers are the most common and popular dermal fillers used for esthetic purpose. ${ }^{1}$ Such dermal fillers are hydrogels, usually composed of $>95 \%$ of water and $0.5-3 \%$ of HA which gives them a gel-like structure. ${ }^{2} \mathrm{HA}$ is a polysaccharide, which is one of the major constituents of the extracellular matrix in vertebrates. It is composed of a repeating disaccharide unit of $(1,4)$-glucuronic acid- $\beta(1,3)$ - $\mathrm{N}$-acetylglucosamine (GlcNAc) linked by glycosidic bonds. This disaccharide pattern is identical among all living organisms. This property makes HA a highly biocompatible molecule in comparison with certain protein-based fillers (eg, collagen), which can exhibit amino acid sequence specificities that maybe recognized by the immune system of the patient.

The major limitation of HA when used as dermal filler is its rapid turnover within the tissues, which is due to the presence of a specific family of enzymes referred to as 
hyaluronidases. To date, several chemical modifications in the HA structure have been described to increase the half-life of HA within tissues. ${ }^{3}$ Most of these modifications try to reduce the access of the hyaluronidases to the polysaccharide polymer by cross-linking the HA chains. Thus, the cross-linked HA hydrogels yield more resistant products to enzymatic degradation than the native HA owing to the formation of bridges, and intermolecular covalent bonds between the HA structure and the cross-linking agent. ${ }^{4-6}$

To date, the chemical cross-linking agents employed to produce cross-linked HA include methacrylamide, ${ }^{7}$ hydrazide, ${ }^{8}$ carbodiimide, ${ }^{9}$ divinyl sulfone, 1,4-butanediol diglycidyl ether (BDDE), and poly (ethylene glycol) diglycidyl ether. $^{10,11}$ BDDE is by far the most commonly employed cross-linking agent nowadays. Although these types of hydrogels have been demonstrated to be safe for decades, the cross-linking agents used are reactive agents that may be cytotoxic and, in certain cases, mutagenic. ${ }^{12}$ Owing to this, their residual presence in the final hydrogel must be highly monitored, and in the case of BDDE, its residual concentration is thought to be safe when it is $<2$ parts per million (ppm). ${ }^{4}$

Several methods allow to detect low residual BDDE concentrations, the degree of cross-linking, and the substitution positions in HA hydrogels, such as gas chromatography, size exclusion chromatography coupled to mass spectrometry (MS), nuclear magnetic resonance (NMR) fluorimetry methodologies, and high-performance liquid chromatography (HPLC) coupled to diode array. ${ }^{13-17}$ The present study describes the detection and characterization of one by-product within the final cross-linked HA hydrogel produced by the reaction between BDDE and HA in alkaline conditions combining both HPLC and liquid chromatography-mass spectroscopy (LC-MS analysis). Since the toxicity of this by-product of BDDE is unknown, we propose that its residual quantification should be determined in a similar manner than the one usually performed for BDDE in the final product.

\section{Materials and methods \\ Materials}

The sodium salt of HA (Shiseido Co., Ltd., Tokyo, Japan) was obtained with a molecular weight of $\sim 1,368,000 \mathrm{Da}$ (Laurent method $)^{18}$ and intrinsic viscosity of $2.20 \mathrm{~m}^{3} / \mathrm{kg}$. For the crosslinking reaction, BDDE ( $\geq 95 \%)$ was purchased from SigmaAldrich Co. (St. Louis, MO, USA). Phosphate-buffered saline $\mathrm{pH} 7.4$ was purchased from Sigma-Aldrich Co. All solvents employed in the LC-MS analysis, acetonitrile, and water, were purchased of HPLC grade quality. Formic acid (98\%) was purchased as reagent grade.

\section{Equipment LC-MS}

All experiments were carried out on a UPLC Acquity system (Waters, Milford, MA, USA) and interfaced to an API 3000 triple quadrupole mass spectrometer equipped with an electrospray ionization source (AB SCIEX, Framingham, MA, USA).

\section{Methods}

\section{Synthesis of cross-linked HA hydrogel}

The synthesis of the cross-linked HA hydrogel was initiated by adding $198 \mathrm{mg}$ of BDDE in a 10\% (w/w) sodium hyaluronate (NaHA) solution in the presence of $1 \%$ of a base (sodium hydroxide, $\mathrm{NaOH}$ ). The final BDDE concentration in the reaction mixture was $9.9 \mathrm{mg} / \mathrm{mL}(0.049 \mathrm{mM})$. Then, the reaction mixture was mixed and homogenized thoroughly, allowing it to proceed for 4 hours at $45^{\circ} \mathrm{C}$. ${ }^{19}$ The reaction $\mathrm{pH}$ was maintained at $\sim 12$.

After this time, the reaction mixture was washed with water and the final HA-BDDE hydrogel was filtrated and diluted with PBS buffer in order to reach a HA concentration ranging from 10 to $25 \mathrm{mg} / \mathrm{mL}$ with a final $\mathrm{pH}$ of 7.4 . To sterilize the produced cross-linked HA hydrogels, all of them have been autoclaved $\left(120^{\circ} \mathrm{C}\right.$ for 20 minutes $)$. The purified BDDE-HA hydrogels were stored at $4^{\circ} \mathrm{C}$ until analysis.

\section{Sample preparation for LC-MS analysis of BDDE}

For the analysis of the BDDE present in the cross-linked HA products, $240 \mathrm{mg}$ of sample was weighed and introduced in a centricon $\left(\right.$ Microcon $^{\circledR}$; Merck Millipore, Billerica, MA, USA; of $0.5 \mathrm{~mL}$ volume) and centrifuged at $10,000 \mathrm{rpm}$ for 10 minutes at room temperature. A total of $20 \mu \mathrm{L}$ of the pulled down liquid were collected and analyzed.

For the analysis of the BDDE standard (Sigma-Aldrich Co) in basic conditions $(1 \%, 0.1 \%$, and $0.01 \% \mathrm{NaOH})$, the liquid sample was diluted at 1:10, 1:100, or up to $1: 1,000,000$ if necessary, with MilliQ deionized water and analyzed.

In the case of the starting material employed in the cross-linking reaction $\left(\mathrm{HA} 2 \%, \mathrm{H}_{2} \mathrm{O}, 1 \% \mathrm{NaOH}\right.$, and 0.049 $\mathrm{mM}$ BDDE), $1 \mathrm{~mL}$ of each sample prepared from these raw materials was analyzed with the same analytical conditions.

In order to establish the specificity of the peaks that appeared in the ionogram, $10 \mu \mathrm{L}$ of 100 parts per billion (ppb) BDDE standard solution (Sigma-Aldrich Co) was added to 20 $\mu \mathrm{L}$ of sample. In this case, the final concentration of standard in each sample was $37 \mathrm{ppb}$. 


\section{Preparation of the BDDE standard curve}

First, a BDDE stock solution was prepared at a concentration of $11,000 \mathrm{mg} / \mathrm{L}(11,000 \mathrm{ppm})$ by diluting $10 \mu \mathrm{L}$ of standard BDDE (Sigma-Aldrich Co) with $990 \mu \mathrm{L}$ of MilliQ water (density $1.1 \mathrm{~g} / \mathrm{mL}$ ). From this solution, a BDDE solution of $110 \mu \mathrm{g} / \mathrm{L}(110 \mathrm{ppb})$ was prepared as an intermediate standard dilution. Then, the intermediate BDDE standard dilution (110 $\mathrm{ppb}$ ) was employed to prepare the standard curve by diluting several times this intermediate dilution to reach the desired concentration of $75,50,25,10$, and $1 \mathrm{ppb}$. As observed in Figure 1, the BDDE standard curve from 1.1 to $110 \mathrm{ppb}$ was found to have a good linearity $\left(\mathrm{R}^{2}>0.99\right)$. Standard curves were repeated in four independent experiments.

\section{Identification and quantitation of BDDE by LC-MS}

To identify and quantify the BDDE present in the cross-linked HA and the BDDE standard in the basic solution, an LC-MS analysis was employed.

The chromatographic separation was achieved on a LUNA $2.5 \mu \mathrm{m} \mathrm{C18}$ (2)-HST column $\left(50 \times 2.0 \mathrm{~mm}^{2}\right.$; Phenomenex, Torrance, CA, USA), which was maintained at room temperature $\left(25^{\circ} \mathrm{C}\right)$ during the analysis. The mobile phase consisted of acetonitrile (solvent $\mathrm{A}$ ) and water with $0.1 \%$ formic acid (solvent B). Mobile phase was eluted in gradient elution mode, and the gradient was as follows: 0 minute, $2 \% \mathrm{~A} ; 1$ minute, $2 \% \mathrm{~A} ; 6$ minutes, $98 \%$ A; 7 minutes, $98 \%$ A; 7.1 minutes, $2 \%$ $\mathrm{A}$; and, 10 minutes, $2 \% \mathrm{~A}$. The run time was 10 minutes, and the sample injection volume was $20 \mu \mathrm{L}$. The retention time for BDDE was $\sim 3.48$ minutes (ranging from 3.43 to 4.14 minutes depending on the experiment). The mobile phase was pumped at a flow rate of $0.25 \mathrm{~mL} / \mathrm{min}$ for LC-MS analysis.

For the BDDE analysis and quantitation by MS, the UPLC system (Waters) was coupled to the API 3000 triple quadrupole mass spectrometer (AB SCIEX) equipped with an electrospray ionization source and analyzed in positive ion mode (ESI+).

From the ion fragmentation analysis carried out on BDDE, it was determined that the fragment having the greatest intensity was the one corresponding to $129.1 \mathrm{Da}$ (Figure 6). Therefore, the quantification was performed in multiple ion monitoring mode (MIM), and the mass transition (mass-to-charge ratio [m/z]) for BDDE were 203.3/129.1 Da. It was also employed full scan (FS) mode and product ion scan (PIS) mode for the LC-MS analysis.

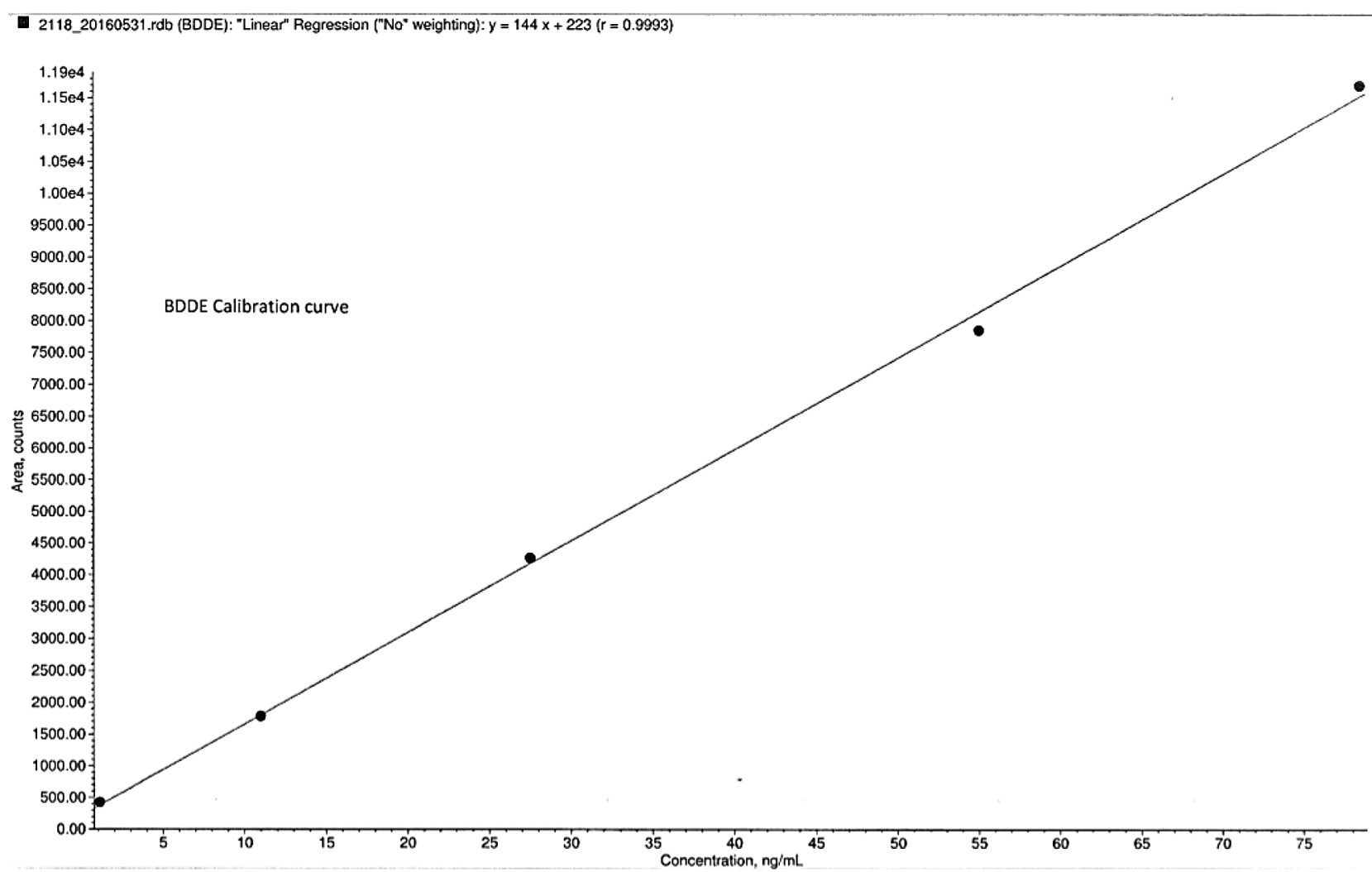

Figure I Standard calibration curve of BDDE obtained by LC-MS analysis, where a good correlation $\left(\mathrm{R}^{2}>0.99\right)$ was observed. Abbreviations: BDDE, I,4-butanediol diglycidyl ether; LC-MS, liquid chromatography and mass spectroscopy. 
In order to verify the specificity of the method, a blank sample (initial mobile phase) was analyzed. No signal was detected in the blank sample for the mass transition 203.3/129.1 Da. Regarding the repeatability of the experiment, 10 injections of the standard at $55 \mathrm{ppb}$ (middle of the calibration curve) were analyzed resulting in a residual standard deviation (RSD) $<5 \%$ (data not shown).

\section{Results}

\section{Analysis and quantification of BDDE in cross-linked HA hydrogels}

The content of residual BDDE was quantified in eight different autoclaved BDDE cross-linked HA hydrogels corresponding to four independent experiments. As described in the "Materials and methods" section, quantification was assessed by the mean of a regression curve of the standard dilutions of BDDE, which corresponds to a unique peak detected at the mass transition for BDDE of 203.3/129.1 Da with a retention time ranging from 3.43 to 4.14 minutes. An example of the chromatogram for the $10 \mathrm{ppb}$ BDDE reference standard is shown in Figure 2. Residual BDDE content of the eight different hydrogels is summarized in Table 1.
The value ranged from 1 to $2.46 \mathrm{ppb}$. The residual BDDE concentrations in the samples were, therefore, acceptable ( $<2 \mathrm{ppm}$ ) for human use.

Surprisingly, the analysis of the LC-MS ion chromatogram revealed the presence of an extra peak at a shorter retention time ranging from 2.73 to 3.29 minutes depending on the experiment for all autoclaved cross-linked HA hydrogel samples analyzed. For example, Figure 3 shows the ion chromatogram of one cross-linked HA sample, where one extra peak appeared at a different retention time of $\sim 2.71$ minutes. The observed relative retention time (RRT) between the new observed peak and the one from the BDDE was found to be 0.79 (Table 1). Since we know that the new observed peak is less retained in the $\mathrm{C} 18$ chromatographic column employed in the LC-MS analysis, the new peak could correspond to a more polar compound than BDDE.

\section{Analysis of the starting material employed}

In order to discard the possibility that the new peak observed could be a contaminant initially present in the raw materials used, these raw materials were also individually subjected

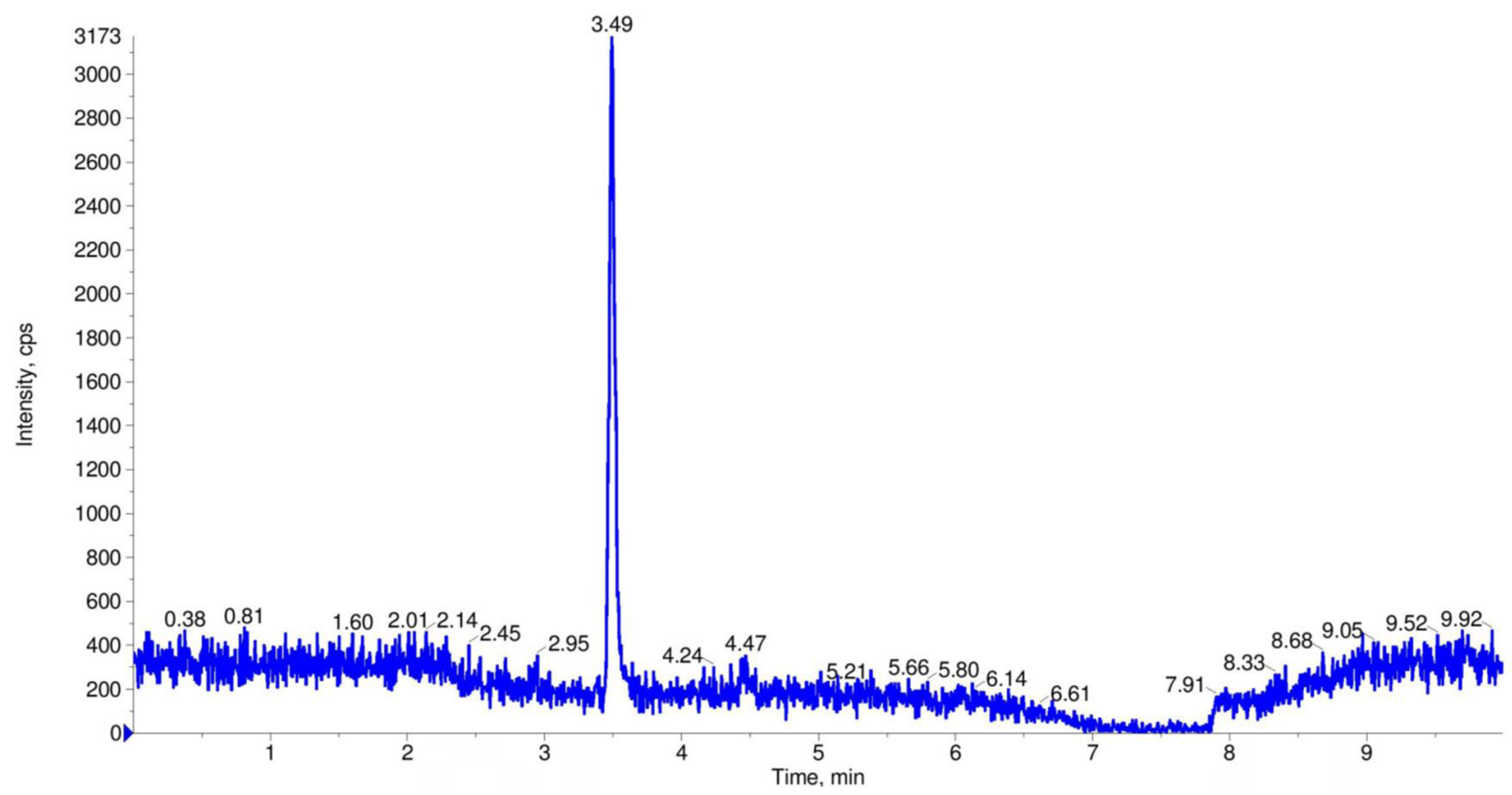

Figure 2 lon chromatogram for the 10 ppb BDDE reference standard (Sigma-Aldrich Co) obtained by LC-MS analysis for the MS (m/z) transition 203.30/I29.10 Da (in positive MRM mode).

Abbreviations: BDDE, I,4-butanediol diglycidyl ether; LC-MS, liquid chromatography and mass spectroscopy; MRM, multiple reaction monitoring; MS, mass; m/z, massto-charge ratio. 
Table I LC-MS analysis results for the mass transition 203.30/I29.10 Da (in positive MRM mode)

\begin{tabular}{llllll}
\hline Sample & $\begin{array}{l}\text { Cross-linked } \mathbf{H A} \\
\text { content in } \mathbf{~} \mathbf{g} \mathbf{m L}\end{array}$ & $\begin{array}{l}\text { BDDE peak } \\
\text { ppb }\end{array}$ & $\begin{array}{l}\text { BDDE peak standard } \\
\text { tR in minutes }\end{array}$ & $\begin{array}{l}\text { New peak }(\mathbf{m g} / \mathbf{m L}) \\
\text { tR in minutes }\end{array}$ & The observed RRT \\
\hline 1 & 20 & 0.13 & 3.43 & 2.73 & 0.79 \\
2 & 10 & 2.11 & 4.14 & 3.29 & 0.79 \\
3 & 12 & 0.41 & 4.14 & 3.29 & 0.79 \\
4 & 14 & 1.44 & 4.14 & 3.29 & 0.79 \\
5 & 17 & 2.46 & 4.14 & 3.29 & 0.79 \\
6 & 25 & $<1$ & 4.08 & 3.20 & 0.78 \\
7 & 10 & $<1$ & 3.50 & 2.79 & 0.79 \\
8 & 25 & $<1$ & 3.50 & 2.79 & 0.79 \\
\hline
\end{tabular}

Notes: Samples I-8 are autoclaved BDDE cross-linked HA hydrogels. Residual BDDE quantity in the hydrogels and BDDE retention times peak are also reported. Finally, the presence of a new peak with different retention time is also reported.

Abbreviations: BDDE, I,4-butanediol diglycidyl ether; HA, hyaluronic acid; MRM, multiple reaction monitoring; tR, retention time; LC-MS, liquid chromatography and mass spectroscopy; RRT, relative retention time.

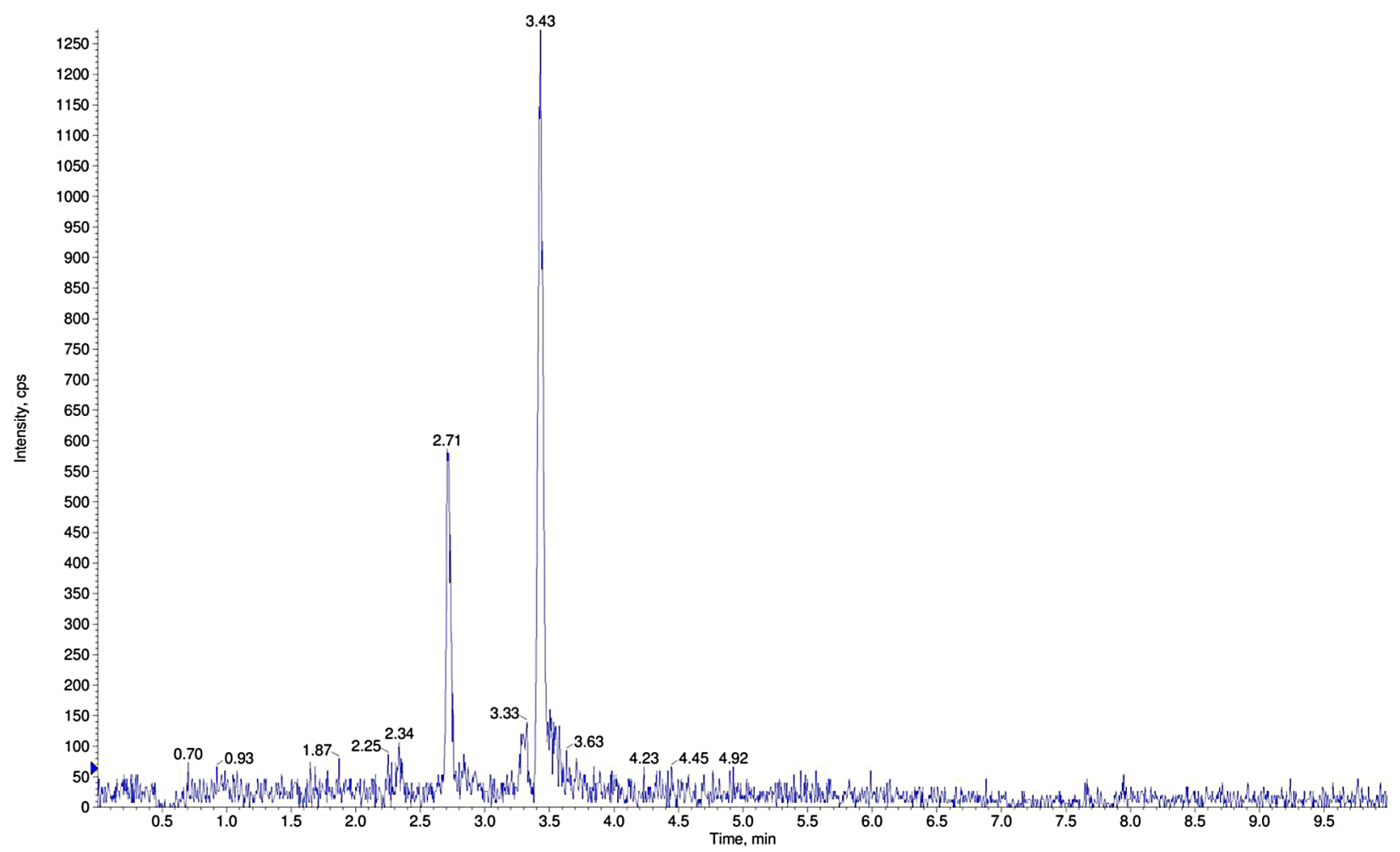

Figure 3 lon chromatogram for the cross-linked HA hydrogel sample obtained by LC-MS (MRM mass transition 203.3/I29.0 Da).

Notes: The new peak with an RRT of 0.79 is observed in this case with a tR of 2.71 minutes.

Abbreviations: HA, hyaluronic acid; LC-MS, liquid chromatography and mass spectroscopy; MRM, multiple reaction monitoring; RRT, relative retention time; tR, retention time.

to analysis using the same LC-MS analytical methodology. The starting materials analyzed include water, a water solution of $2 \% \mathrm{NaHA}, 1 \% \mathrm{NaOH}$ in aqueous solution, and BDDE at the same concentration used in the cross-link reaction. The ion chromatograms of the starting materials employed did not present any compound or peak with a retention time corresponding with the new peak observed. This fact discards the idea that not only the starting material could present any compound or substance that may interfere with the analytical procedure but also there is no sign of any possible cross-contamination with other laboratory products. The concentration values obtained after the LC-MS analysis regarding BDDE and the new peak are shown in Table 2 (samples 1-4) and in the ion chromatograms from Figure 4.

\section{Analysis of the BDDE in the presence and absence of $\mathrm{NaOH}$}

Conditions that lead to the formation of the new peak were investigated. In order to study how the reaction conditions employed to produce the cross-linked HA hydrogel affect 
Table 2 LC-MS analysis results for the mass transition 203.30/129.10 Da (in positive MRM mode)

\begin{tabular}{llll}
\hline Sample & Sample identity & $\begin{array}{l}\text { New peak } \\
(\mathbf{m g} / \mathbf{m L})\end{array}$ & $\begin{array}{l}\text { BDDE } \\
\text { peak }(\mathbf{m g} / \mathbf{m L})\end{array}$ \\
\hline $\mathrm{I}$ & $\mathrm{H}_{2} \mathrm{O}$ & 0 & 0 \\
2 & $\mathrm{HA}$ at $2 \%$ & 0 & 0 \\
3 & $\mathrm{\%} \mathrm{NaOH}$ in $\mathrm{H}_{2} \mathrm{O}$ & 0 & 0 \\
4 & BDDE in $\mathrm{H}_{2} \mathrm{O}$ & 0 & 10.15 \\
\hline
\end{tabular}

Notes: Samples 1-4 correspond to the raw materials used to produce the autoclaved BDDE cross-linked HA hydrogels. These samples were not autoclaved. Abbreviations: BDDE, I,4-butanediol diglycidyl ether; HA, hyaluronic acid; LC-MS, liquid chromatography and mass spectroscopy; MRM, multiple reaction monitoring.

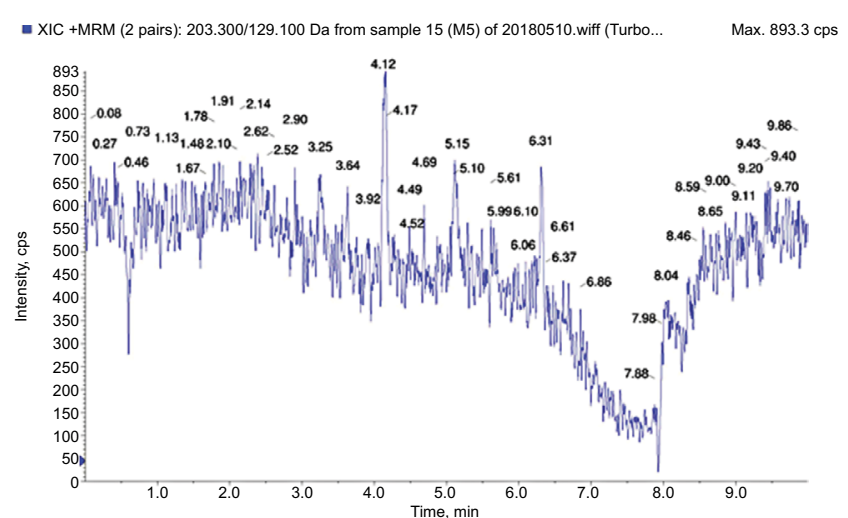

- XIC +MRM (2 pairs): 203.300/129.100 Da from sample 16 (M6) of 20180510.wiff (Turbo... Max. 735.0cps
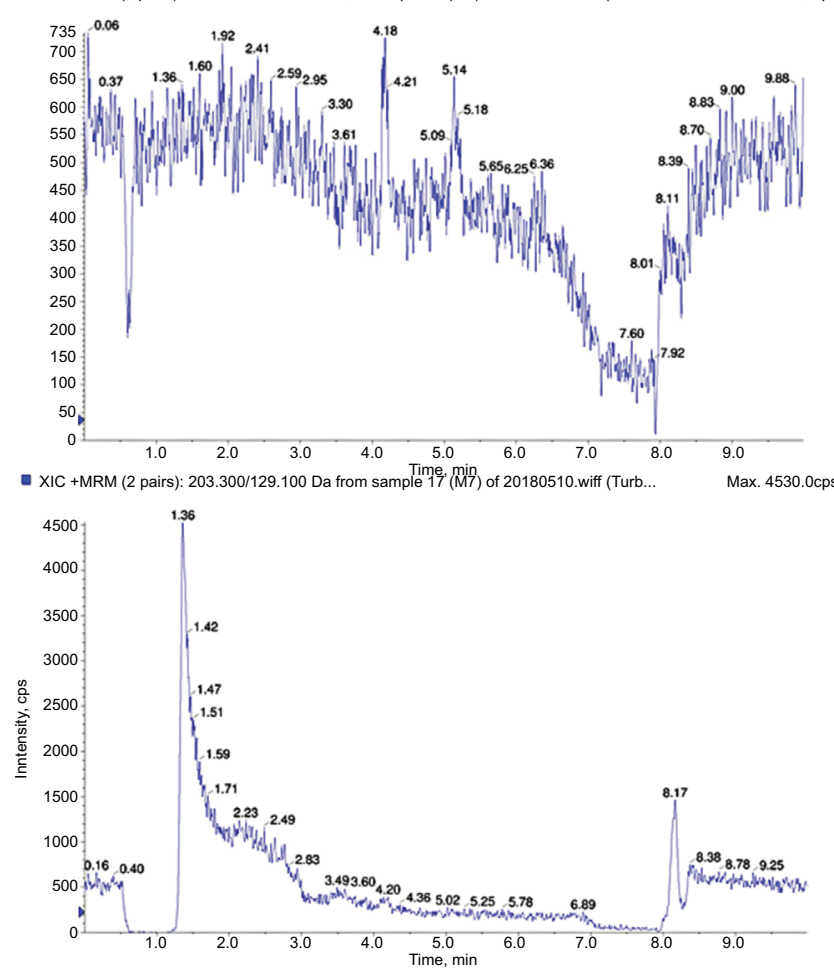

Figure 4 LC-MS chromatogram for the samples corresponding to the raw materials employed in the cross-linking reaction of the HA with BDDE.

Notes: All of them were assayed at same concentration and proportions as employed to carry out the cross-linking reaction. Numerical correspondence of the raw materials analyzed regarding the chromatograms: (I) water, (2) $2 \% \mathrm{HA}$ in water, (3) $1 \% \mathrm{NaOH}$ in aqueous solution. The LC-MS analysis was carried out for the mass transition 203.30/129.10 Da (in positive MRM mode).

Abbreviations: BDDE, I,4-butanediol diglycidyl ether; HA, hyaluronic acid; LC-MS, liquid chromatography and mass spectroscopy; MRM, multiple reaction monitoring. the reactivity of the BDDE cross-linker leading to the formation of the new peak (possible by-product), different assays were carried out. In these assays, we studied and analyzed the final BDDE cross-linker after treating it with different $\mathrm{NaOH}$ concentrations $(0 \%, 1 \%, 0.1 \%$, and $0.01 \%)$ in aqueous media followed or not by an autoclaving procedure to simulate the same conditions as that of the one performed to produce the cross-linked HA hydrogel. The samples were analyzed by LC-MS for the mass transition 203.30/129.10 $\mathrm{Da}$ as described in the "Materials and methods" section. The concentrations found for BDDE and the new peak were calculated, and the results are presented in Table 3. The new peak was not detected in samples that were not autoclaved, independently of the presence of $\mathrm{NaOH}$ in the solution (samples 1-4, Table 3). In the case of autoclaved samples, the new peak was detected only in conditions presenting $\mathrm{NaOH}$ in the solution, and the formation of the peak appeared to be dependent on the $\mathrm{NaOH}$ concentration in the solution (samples 5-8, Table 3) $($ RRT $=0.79)$. The example of ion chromatogram, presenting two autoclaved samples in the presence or not of NAOH is given in Figure 5.

In all autoclaved samples, BDDE concentration was greatly reduced (up to 16.6 folds) (samples $5-8$, Table 2) with or without $\mathrm{NaOH}$. This decrease in BDDE concentration maybe explained by the fact that at high temperatures, water can act as a base (nucleophile) opening the epoxide ring of BDDE forming a 1,2-diol compound, which would not have the same monoisotopic mass than the BDDE and therefore would not be detected by the LC-MS for the mass transition 203.30/129.10 Da.

Finally, these experiments suggest that the creation of the new peak is dependent on the presence of BDDE, $\mathrm{NAOH}$, and the autoclaving process but is independent of the HA.

\section{Mass characterization of the new peak}

The new peak found at $\sim 2.71$ minutes of retention time was then characterized by LC-MS. For this purpose, BDDE ( 9.9 $\mathrm{mg} / \mathrm{mL}$ ) was incubated in the aqueous solution of $1 \% \mathrm{NaOH}$ and autoclaved. In Table 4, the characteristics of the new peak is compared to the known reference peak of BDDE (retention time about 3.47 minutes). From the analysis of the ion fragmentation analysis carried out for both peaks, it can be concluded that the peak at 2.72 minutes of retention time shows the same fragments as that of the BDDE peak, but with differences in their intensity (Figure 6). A more intense peak was observed after fragmentation at a mass of $147 \mathrm{Da}$ for the peak corresponding to the 2.72 minutes 
Table 3 LC-MS analysis results for the mass transition 203.30/I29.10 Da (in positive MRM mode)

\begin{tabular}{llll}
\hline Sample & Sample identity & New peak $(\mathbf{m g} / \mathbf{m L})$ & BDDE peak $(\mathbf{m g} / \mathbf{m L})$ \\
\hline $\mathrm{I}$ & BDDE in $\mathrm{H}_{2} \mathrm{O}$ & 0 & 10.15 \\
2 & BDDE in I\% $\mathrm{NaOH}$ aqueous solution & 0 & 0.14 \\
3 & BDDE in $0.1 \% \mathrm{NaOH}$ aqueous solution & 0 & 6.97 \\
4 & BDDE in $0.01 \% \mathrm{NaOH}$ aqueous solution & 0 & 9.72 \\
5 & BDDE in $\mathrm{H}_{2} \mathrm{O}$ autoclaved & 0 & 0.61 \\
6 & BDDE in $1 \% \mathrm{NaOH}$ aqueous solution autoclaved & $5.47 \times 10^{-6}$ & $1.12 \times 10^{-6}$ \\
7 & BDDE in $0.1 \% \mathrm{NaOH}$ aqueous solution autoclaved & $0.98 \times 10^{-3}$ & $1.98 \times 10^{-3}$ \\
8 & BDDE in $0.01 \% \mathrm{NaOH}$ aqueous solution autoclaved & 0 & 0.44 \\
\hline
\end{tabular}

Note: Samples I-4 were not autoclaved, whereas samples 5-8 were autoclaved.

Abbreviations: BDDE, I,4-butanediol diglycidyl ether; LC-MS, liquid chromatography and mass spectroscopy; MRM, multiple reaction monitoring.

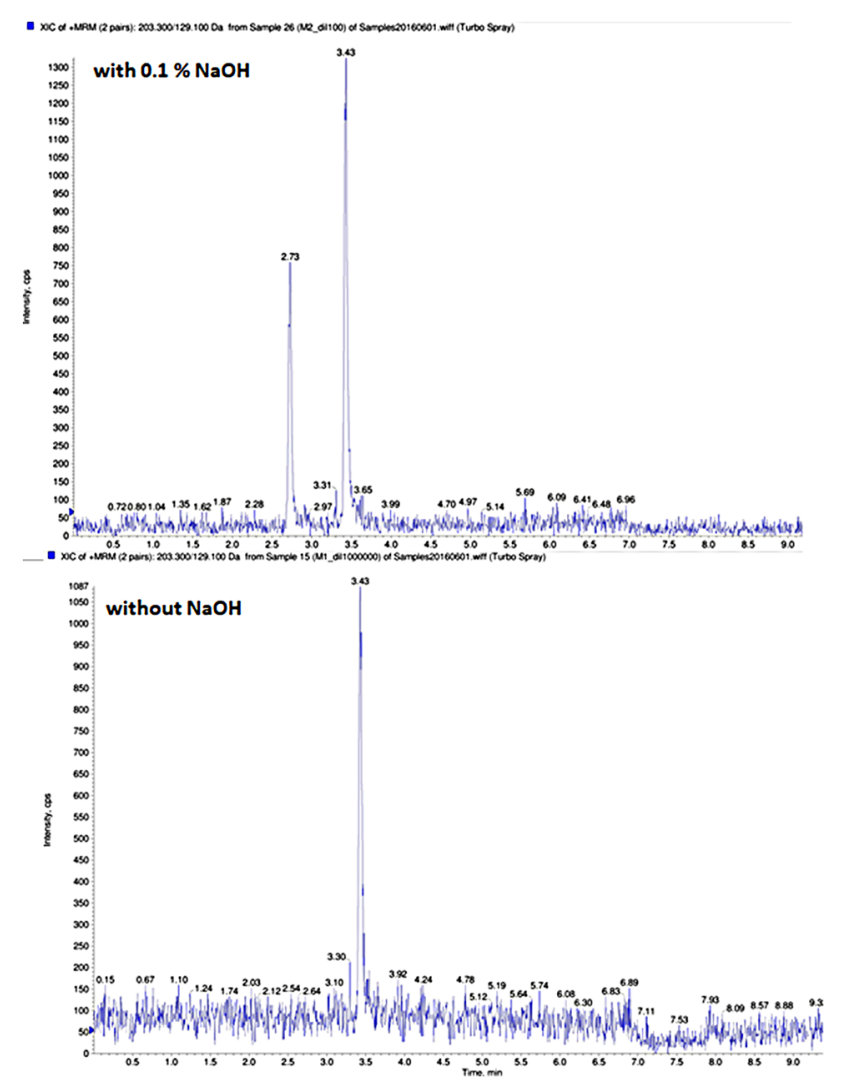

Figure 5 Chromatogram for the BDDE studied in the presence and absence of $\mathrm{NaOH}$.

Notes: Chromatogram at the top: the sample was treated with $0.1 \% \mathrm{NaOH}$ in aqueous media and autoclaved $\left(120^{\circ} \mathrm{C}\right.$ for 20 minutes). Chromatogram at the bottom: the sample was not treated with $\mathrm{NaOH}$ but was autoclaved in the same conditions. The analysis was performed by LC-MS for the mass transition 203.30/I29.10 Da (in positive MRM mode).

Abbreviations: BDDE, I,4-butanediol diglycidyl ether; LC-MS, liquid chromatography and mass spectroscopy; MRM, multiple reaction monitoring.

retention time (PIS). At the BDDE concentrations employed in this assay $(9.9 \mathrm{mg} / \mathrm{mL})$, a different absorbance pattern in the ultraviolet spectra was also observed (UV, $\lambda=200 \mathrm{~nm}$ ) after their chromatographic separation (Figure 7). The peak presenting the retention time of 2.71 minutes is still visible at $200 \mathrm{~nm}$ detection while the BDDE peak cannot be observed in the chromatogram at the same conditions.
Table 4 Results of the characterization of the new peak with a retention time of $\sim 2.71$ minutes and the BDDE peak found with a retention time of 3.47 minutes

\begin{tabular}{lll}
\hline Sample & New peak & BDDE peak \\
\hline $\mathrm{m} / \mathrm{z}$ & $203.3 \mathrm{Da}$ & $203.3 \mathrm{Da}$ \\
Retention time & $2.7 \mathrm{I}$ minutes & 3.47 minutes \\
Most intense fragments & $\mid 47.0>73.2>130.7$ & $\mid 28.9>72.8>146.9$ \\
Absorbance at $200 \mathrm{~nm}$ & Yes & No \\
MRM most intense peak & 147.0 & 128.9 \\
\hline
\end{tabular}

Notes: To obtain these results, LC-MS and HPLC analyses (MRM and PIS) were performed for both peaks. For the HPLC analysis, an UV detection at $200 \mathrm{~nm}$ of wavelength was employed.

Abbreviations: BDDE, I,4-butanediol diglycidyl ether; HPLC, high performance liquid chromatography; LC-MS, liquid chromatography and mass spectroscopy; MRM, multiple reaction monitoring; $\mathrm{m} / \mathrm{z}$, mass-to-charge ratio; PIS, product ion scan; UV, ultraviolet.

\section{Discussion}

The concentration of the residual BDDE after LC-MS quantification was observed to be $<2 \mathrm{ppm}$ in all of the cross-linked HA hydrogel produced, but a new unknown peak appeared in the analysis. This new peak does not correspond with the BDDE standard which was also analyzed for the same mass transition (MRM transition 203.30/129.10 Da) performed in positive MRM mode. Normally, other analytical methodologies like chromatography are used as limit tests in order to detect BDDE in hydrogels, but with a maximum limit of detection (LOD) a little lower than $2 \mathrm{ppm}$. On the other hand, NMR and MS were employed so far to characterize the degree of cross-linking and/or the modification of HA in fragments of the saccharide units of the cross-linked HA product. The purpose of these techniques was never quantifying the residual BDDE detection at such lower concentrations as we described (LOD of our LC-MS method $=10 \mathrm{ppb}$ ) in this article.

The results of this study suggest that the basic reaction conditions of the reaction for the synthesis of the crosslinked HA hydrogel (in the presence of $1 \% \mathrm{NaOH}$ ) followed by autoclaving procedures could lead to the formation of a 


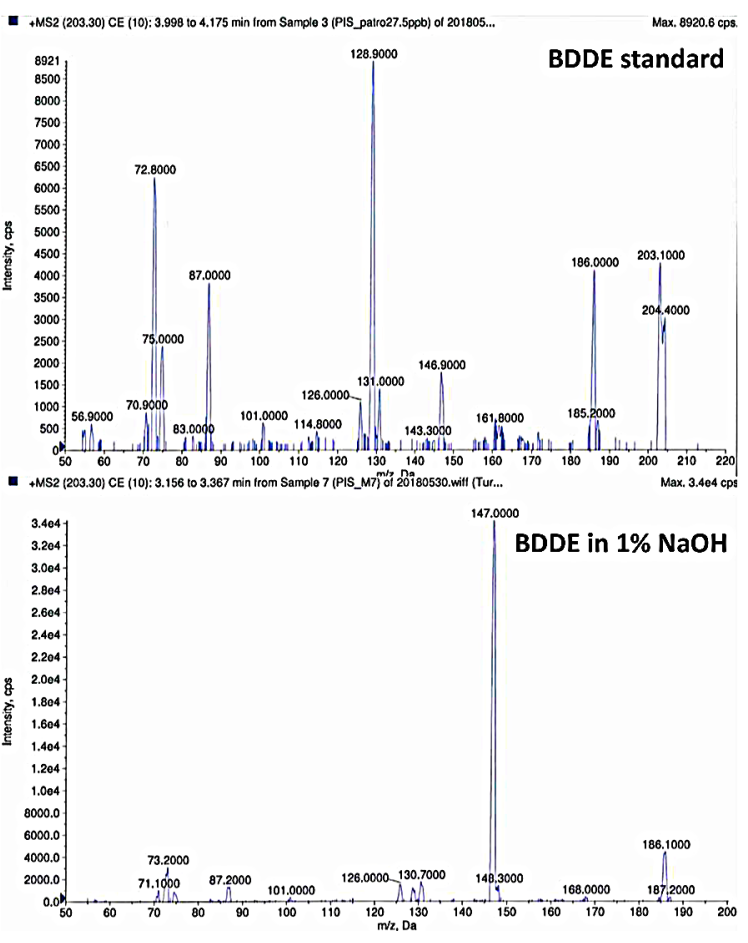

Figure 6 Mass spectra of the fragmented parent ion of $203.20 \mathrm{Da}$. Notes: Mass fragments were obtained by LC-MS analysis (PIS). Chromatogram at the top: the mass spectra of the fragments for the BDDE standard sample. Chromatogram at the bottom: mass spectra of the new peak detected (RRT of 0.79 related to BDDE peak) from the treatment of BDDE in a $1 \% \mathrm{NaOH}$ solution and autoclaved.

Abbreviations: BDDE, I,4-butanediol diglycidyl ether; LC-MS, liquid chromatography and mass spectroscopy; MRM, multiple reaction monitoring; PIS, product ion scan; RRT, relative retention time.

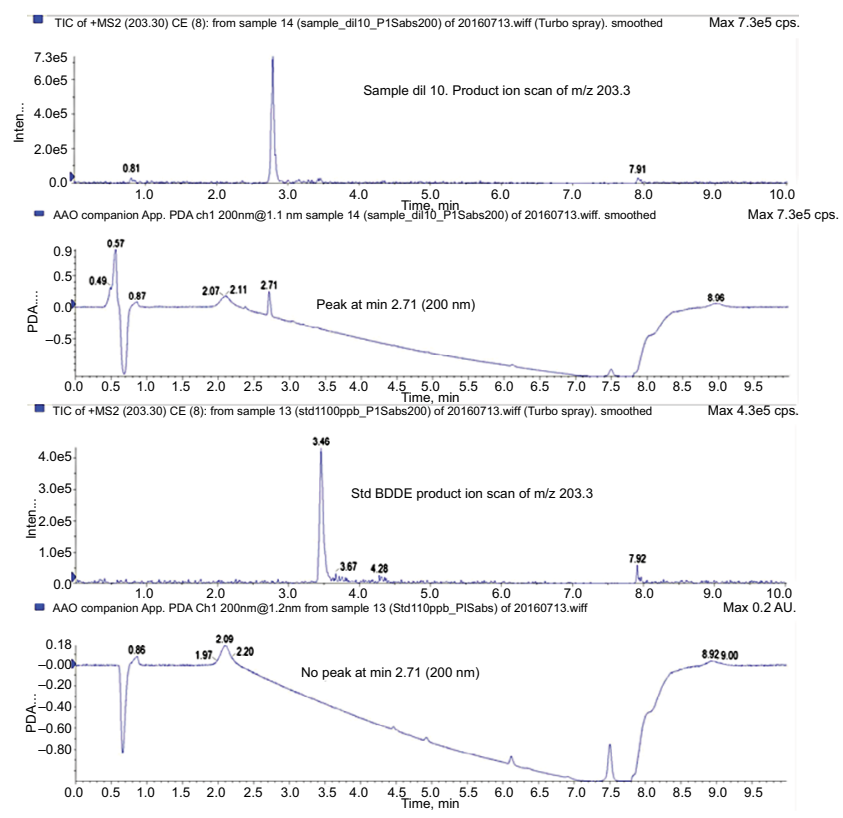

Figure 7 The ion chromatogram for the parent ion of $203.30 \mathrm{Da}$, and the UV detection at $200 \mathrm{~nm}$ obtained for (A) the new peak with a retention time of 2.7I minutes and $(B)$ the BDDE reference standard peak at 3.46 minutes. Abbreviations: BDDE, I,4-butanediol diglycidyl ether; UV, ultraviolet.
BDDE by-product that can be detected at a different retention time in the ion chromatograms that were analyzed. The new peak observed was identified in the ion chromatogram with an RRT of 0.79 relative to the BDDE peak standard.

When analyzing the starting materials including BDDE, water, $\mathrm{NaOH}$ solution at $1 \%$, and $\mathrm{NaHA}$ solution at $2 \%$ independently, the possible presence of any contaminant in the samples that could be responsible for the detection of the new peak was discarded.

The studies in basic conditions $(\mathrm{NaOH})$ demonstrated that this new peak was also observed in the absence of HA and appeared exclusively when autoclaving process was performed $\left(120^{\circ} \mathrm{C}\right.$ for 20 minutes). The appearance of this new peak was favored by the increasing concentration of $\mathrm{NaOH}$ in the BDDE solution. On the contrary, when the BDDE samples lacking the presence of the base $(\mathrm{NaOH})$ were autoclaved, the new peak was never observed in the analyses.

The decrease of BDDE owing to autoclaving in aqueous media (water) and in the absence of a base is explained by the fact that water can act as a nucleophile at high temperatures opening the epoxide ring and forming a 1,2-diol-based product. Because of the mass transitions analyzed by LC-MS (203.20/129.10 and 203.3/147.00 Da), the possibility of detecting the 1,2-diol-based compound was not possible.

The mass studies demonstrated that this new peak corresponded with a compound with the same monoisotopic mass than the BDDE reference (202.120514 Da), but with a more polar nature owing to its shorter retention time compared with BDDE in the LC-MS studied conditions. The RRT found for this peak was 0.79 relative to BDDE for the mass transition 203.20/129.10 Da (positive MRM mode). These new peaks have been demonstrated to have absorbance in the UV spectra $(\lambda=200 \mathrm{~nm})$. The fact that this possible BDDE by-product has a higher detection at $200 \mathrm{~nm}$ suggests that the new compound would be neither a BDDE isomer (enantiomer) nor any other compound having an oxirane (an epoxide group) in its structure.

On the other hand, the parental ion fragmentation with a mass of 203.20 Da fragmentation for BDDE and the new suspected compound formed presented a similar fragmentation pattern than BDDE but differed in their intensity. However, different higher intense peaks were observed with a $\mathrm{m} / \mathrm{z}$ of 129.1 and 147.0 Da for BDDE and the new peak, respectively.

Once the possibility of being a BDDE enantiomer has been discarded and knowing that the 1,2-diol product formed by the epoxide ring opening was not the purpose of our analyses, a proposed explanation could be the formation of one of the tautomers shown in Figure 8. These proposed compounds would exactly have the same analyzed monoisotopic mass as 


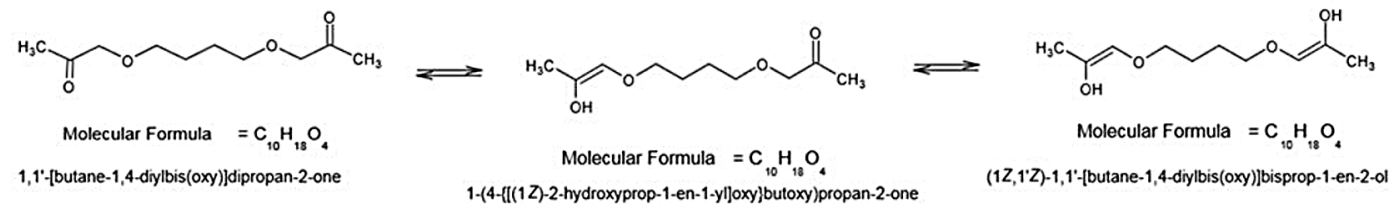

Figure 8 The three tautomeric forms proposed as BDDE by-products occurring during the cross-linking reaction of BDDE with HA, but also without HA, and catalyzed by $\mathrm{NaOH}$ and at high temperature $\left(121^{\circ} \mathrm{C}\right.$ for 20 minutes).

Abbreviations: BDDE, I,4-butanediol diglycidyl ether; HA, hyaluronic acid.

the BDDE cross-linker, without differing in mass from the same parental ion of 203.20 Da of BDDE.

The reaction proposed for the formation of these tautomers would be a reaction catalyzed in the basic media employed in the cross-linking reaction and favored by the high temperature treatment for sterilization of dermal fillers. This reaction would lead to the irreversible loss of the epoxy ring from its structure. However, the reaction mechanism that led to this by-product has not been elucidated yet, but it is possible that the base may act as a catalyst at high temperatures, existing in an equilibrium at $\mathrm{pH} 7.4$ (the final $\mathrm{pH}$ of the HA hydrogels produced) between these three forms presented in Figure 8, where the most favorable tautomer will prevail between them.

\section{Conclusion}

Our study demonstrated the formation of a by-product in the cross-linking reaction of BDDE with HA to form cross-linked HA hydrogels (HA-BDDE) which is formed in the presence of a base following autoclaving procedure.

As the toxicity of this by-product of BDDE is unknown, we propose that its residual quantification should be determined in a similar manner as that usually performed for BDDE in the final product. Since autoclaving procedures are usually employed for the sterilization of dermal fillers, we believe that this detected product should be taken into account.

\section{Acknowledgments}

The authors would like to thank Dr Isidre Casals for his kind support and advise in understanding the analytical results, and the collaboration made by his group in this research. His group has kindly analyzed our cross-linked HA samples and also characterized and analyzed the BDDE present in our different proposed assays (Universitat de Barcelona, Unitat Tècniques Separatives, Barcelona, Spain).

\section{Disclosure}

The authors report no conflicts of interest in this work.

\section{References}

1. Brandt FS, Cazzaniga A. Hyaluronic acid gel fillers in the management of facial aging. Clin Interv Aging. 2008;3(1):153.
2. Enas MA. Hydrogel: preparation, characterization, and applications JAdv Res Cairo Univ. 2015;6:105-121.

3. Jeon O, Song SJ, Lee K-J, et al. Mechanical properties and degradation behaviors of hyaluronic acid hydrogels cross-linked at various crosslinking densities. Carbohydr Polym. 2007;70(3):251-257.

4. de Boulle K, Glogau R, Kono T, et al. A review of the metabolism of 1,4-butanediol diglycidyl ether-crosslinked hyaluronic acid dermal fillers. Dermatol Surg. 2013;39(12):1758-1766.

5. Burdick JA, Prestwich GD. Hyaluronic acid hydrogels for biomedical applications. Adv Mater. 2011;23(12):H41-H56.

6. Yeom J, Bhang SH, Kim BS, et al. Effect of cross-linking reagents for hyaluronic acid hydrogel dermal fillers on tissue augmentation and regeneration. Bioconjug Chem. 2010;21(2):240-247.

7. Segura T, Anderson BC, Chung PH, Webber RE, Shull KR, Shea LD. Crosslinked hyaluronic acid hydrogels: a strategy to functionalize and pattern. Biomaterials. 2005;26(4):359-371.

8. Prestwich GD, Marecak DM, Marecek JF, Vercruysse KP, Ziebell MR Controlled chemical modification of hyaluronic acid: synthesis, applications, and biodegradation of hydrazide derivatives. $J$ Control Release. 1998;53(1-3):93-103.

9. Lai J-Y. Solvent composition is critical for carbodiimide crosslinking of hyaluronic acid as an ophthalmic biomaterial. Materials. 2012;5(10):1986-2002.

10. La Gatta A, Schiraldi C, Papa A, et al. Hyaluronan scaffolds via diglycidyl ether crosslinking: toward improvements in composition and performance. Carbohydr Polym. 2013;96(2):536-544.

11. Schanté CE, Zuber G, Herlin C, Vandamme TF. Chemical modifications of hyaluronic acid for the synthesis of derivatives for a broad range of biomedical applications. Carbohydr Polym. 2011;85(3): 469-489.

12. Foureman P, Mason JM, Valencia R, Zimmering S. Chemical mutagenesis testing in Drosophila. IX. Results of 50 coded compounds tested for the National Toxicology Program. Environ Mol Mutagen. 1994;23(1):51-63.

13. Hai F, Ge Z, Yu-Yun X. Determination of free 1,4-butanediol diglycidyl ether in cross-linked hyaluronic acid gel by fluorospectrophotometry. Chin J Pharm Anal. 2012;32(4):654-657.

14. Wende FJ, Gohil S, Nord LI, Helander Kenne A, Sandström C. 1D NMR methods for determination of degree of cross-linking and BDDE substitution positions in HA hydrogels. Carbohydr Polym. 2017;157:1525-1530.

15. Yang B, Guo X, Zang H, Liu J. Determination of modification degree in BDDE-modified hyaluronic acid hydrogel by SEC/MS. Carbohydr Polym. 2015;131:233-239.

16. Wende FJ, Gohil S, Mojarradi H, et al. Determination of substitution positions in hyaluronic acid hydrogels using NMR and MS based methods. Carbohydr Polym. 2016;136:1348-1357.

17. Al-Sibani M, Al-Harrasi A, Neubert RHH. Evaluation of in-vitro degradation rate of hyaluronic acid-based hydrogel cross-linked with 1,4-butanediol diglycidyl ether (BDDE) using RP-HPLC and UV-Vis spectroscopy. J Drug Deliv Sci Technol. 2015;29:24-30.

18. Laurent TC. An early look at macromolecular crowding. Biophys Chem. 1995;57(1):7-14.

19. Mälson T, Lindqvist BL, inventors; Pharmacia AB, assignee. Gel of crosslinked hyaluronic acid for use as a vitreous humor substitute. United States patent US WO86/00079 (US patent 4). 1987 Dec 29. 


\section{Publish your work in this journal}

Medical Devices: Evidence and Research is an international, peerreviewed, open access journal that focuses on the evidence, technology, research, and expert opinion supporting the use and application of medical devices in the diagnosis, monitoring, treatment and management of clinical conditions and physiological processes. The identification of novel devices and optimal use of existing devices which will lead to improved clinical outcomes and more effective patient management and safety is a key feature. The manuscript management system is completely online and includes a quick and fair peer-review system. Visit http://www. dovepress.com/testimonials.php to read real quotes from authors.

Submit your manuscript here: https://www.dovepress.com/medical-devices-evidence-and-research-journal 